\title{
Effect of a Non-Zero Velocity of the Motion Frame on the Kinetic Energy of a Free Particle Examined with the Aid of the Relativistic Mechanics
}

\author{
Stanisław Olszewski \\ Institute of Physical Chemistry, Polish Academy of Sciences, Warsaw, Poland \\ Email: solszewski@ichf.edu.pl
}

\begin{abstract}
How to cite this paper: Olszewski, S. (2021) Effect of a Non-Zero Velocity of the Motion Frame on the Kinetic Energy of a Free Particle Examined with the Aid of the Relativistic Mechanics. World Journal of Mechanics, 11, 1-6.

https://doi.org/10.4236/wjm.2021.111001
\end{abstract}

Received: November 17, 2020

Accepted: January 23, 2021

Published: January 26, 2021

Copyright (c) 2021 by author(s) and Scientific Research Publishing Inc.

This work is licensed under the Creative Commons Attribution International License (CC BY 4.0).

http://creativecommons.org/licenses/by/4.0/

(c) (i) Open Access

\begin{abstract}
The paper examines the change of the relativistic kinetic energy of a free particle due to the velocity change of the motion frame in a special case when this reduction leads to the kinetic energy equal to zero. The difference of velocities gives a functional dependent solely on the velocity frame and original velocity of the particle. An analysis applied to the functional gives simple formulae for the extremal values of the mentioned velocity parameters. In the next step, solutions of the equation presented with the functional provide us with the velocities necessary for the vanishing property of the kinetic energy. A characteristic point is that a condition of the velocity of the motion frame smaller than the velocity of light is obtained directly in the applied formalism. This property holds with no reference done to the well-known postulate of the dominant value of the light velocity entering the relativity theory.
\end{abstract}

\section{Keywords}

Relativistic Kinetic Energy, Reduction of the Particle Velocity due to the Velocity of the Motion Frame

\section{Introduction}

Functional dependencies representing the differences between mechanical parameters entering the relativity theory seem to be rather seldom discussed. The aim of the paper is to get an insight into the problem how the kinetic energy of a free particle is influenced by the speed of the frame characteristic for the examined system. If the speed of the frame is $w$, and it has the same direction as the original particle velocity $u$, the well-known formula for the resulted change of $u$ due to 
$w$ is represented by the velocity [1]

$$
u^{\prime}=\frac{u-w}{1-\frac{u w}{c^{2}}} .
$$

Here we assumed - for the sake simplicity - that $u, w$ and $u^{\prime}$ are the velocities directed along the same Cartesian coordinate.

The change of the relativistic kinetic energy which is the effect of modification of $u$ into $u^{\prime}$ becomes $[2,3]$

$$
\begin{aligned}
\Delta E_{\mathrm{kin}} & =E_{\mathrm{kin}}\left(u^{\prime}\right)-E_{\mathrm{kin}}(u)=m_{0} c^{2}\left(\frac{1}{\sqrt{1-\frac{u^{\prime 2}}{c^{2}}}}-\frac{1}{\sqrt{1-\frac{u^{2}}{c^{2}}}}\right) \\
& =m_{0} c^{2}\left[\frac{1}{2}\left(\frac{u^{\prime 2}}{c^{2}}-\frac{u^{2}}{c^{2}}\right)+\frac{3}{8}\left(\frac{u^{\prime 4}}{c^{4}}-\frac{u^{4}}{c^{4}}\right)+\cdots\right] \\
& =\frac{m_{0}}{2}\left(u^{\prime 2}-u^{2}\right)\left[1+\frac{3}{4 c^{2}}\left(u^{\prime 2}+u^{2}\right)+\cdots\right]
\end{aligned}
$$

where $m_{0}$ is the rest mass of the particle, $c$ is the speed of light. The difference (2), expanded into the power series, represents a sufficient accuracy on condition

$$
u^{\prime 2}<c^{2}
$$

and

$$
u^{2}<c^{2}
$$

In a simplest case, when the velocity $w$ of the moving frame of a system is assumed to be a constant, and a similar property applies to the velocity $u$ of the moving particle, only two chosen parameters, i.e. $w$ and $u$, do influence the relativistic properties of the moving body. A question then arises: which are the properties of the kinetic energy interval defined in (2)?

From the power expansion (2), we see that successive terms dependent on $c$ rapidly decrease, so we can reject the terms

$$
c^{-4}, c^{-6}, c^{-8}, \ldots
$$

etc., as being very small. In effect, our study can be limited to only few terms entering (2).

But a search for the properties of the difference (2) can be much simplified if we note that all these terms are proportional to the difference

$$
u^{\prime 2}-u^{2}
$$

so our task can be reduced to examine mainly the situation when (5) becomes zero. 


\section{The Difference of the Velocity Squares in (5) Put Equal to Zero and the Behaviour of Its Parameters}

The difference (5) can be represented as follows:

$$
\begin{aligned}
u^{\prime 2}-u^{2} & =\frac{(u-w)^{2}}{\left(1-\frac{u w}{c^{2}}\right)^{2}}-u^{2}=\frac{(u-w)^{2}-u^{2}\left(1-\frac{u w}{c^{2}}\right)^{2}}{\left(1-\frac{u w}{c^{2}}\right)^{2}} \\
& =\frac{1}{\left(1-\frac{u w}{c^{2}}\right)^{2}}\left[u-w-u\left(1-\frac{u w}{c^{2}}\right)\right]\left[u-w+u\left(1-\frac{u w}{c^{2}}\right)\right] \\
& =\frac{-w\left(1-\frac{u^{2}}{c^{2}}\right)}{\left(1-\frac{u w}{c^{2}}\right)^{2}}\left(-\frac{u^{2} w}{c^{2}}+2 \frac{u c}{c}-w\right) \\
& =\frac{w\left(1-\frac{u^{2}}{c^{2}}\right)}{\left(1-\frac{u w}{c^{2}}\right)^{2}}\left(x^{2} w-2 c x+w\right)=0 .
\end{aligned}
$$

Only the last bracket term in (6) is important because of

$$
u, w<c .
$$

Moreover we defined

$$
x=u / c .
$$

The solution of the second-order algebraic equation given in (6) is simple. We have

$$
\Delta=4 c^{2}-4 w^{2}=4 c^{2}\left(1-\frac{w^{2}}{c^{2}}\right)
$$

SO

$$
\Delta^{1 / 2}=2 c\left(1-\frac{w^{2}}{c^{2}}\right)^{1 / 2}
$$

and

$$
x_{1,2}=\frac{2 c \pm \Delta^{1 / 2}}{2 w}=\frac{c}{w}\left[1 \pm\left(1-\frac{w^{2}}{c^{2}}\right)^{1 / 2}\right] .
$$

Another set of solutions is obtained when $-w$ in (1) and (6) is replaced by $w$. In this case we have

$$
u^{\prime}=\frac{u+w}{1+\frac{u w}{c^{2}}}
$$

and our task becomes the solution of the equation

$$
\frac{(u+w)^{2}}{\left(1+\frac{u w}{c^{2}}\right)^{2}}-u^{2}=0 .
$$

We obtain in this case from the numerator:

$$
\begin{aligned}
(u+w)^{2}-u^{2}\left(1+\frac{u w}{c^{2}}\right)^{2} & =\left(w-\frac{u^{2} w}{c^{2}}\right)\left(2 u+w+\frac{u^{2} w}{c^{2}}\right) \\
& =w\left(1-\frac{u^{2}}{c^{2}}\right)\left(x^{2} w+2 x c+w\right)=0
\end{aligned}
$$


where the definition of $x$ in (8) is taken into account. The solution of the algebraic equation entering (14) is given by

$$
\begin{aligned}
& \Delta=4\left(c^{2}-w^{2}\right)=4 c^{2}\left(1-\frac{w^{2}}{c^{2}}\right), \\
& \Delta^{1 / 2}=2 c\left(1-\frac{w^{2}}{c^{2}}\right)^{1 / 2}
\end{aligned}
$$

so

$$
x_{1,2}=\frac{-2 c \pm 2 c\left(1-\frac{w^{2}}{c^{2}}\right)^{1 / 2}}{2 w}=-\frac{c}{w}\left[1 \mp\left(1-\frac{w^{2}}{c^{2}}\right)^{1 / 2}\right] .
$$

\section{Some Extremal Properties of the Difference Entering the Equations (6) and (13)}

By denoting

$$
F_{1}(x)=x^{2} w-2 c x+w
$$

we obtain from its first derivative the equation

$$
F_{1}^{\prime}(x)=2 x w-2 c=0
$$

from which

$$
x=x_{\min }=\frac{c}{w}
$$

SO

$$
F_{1}\left(x_{\min }\right)=w\left(\frac{c}{w}\right)^{2}-2 c\left(\frac{c}{w}\right)+w=-\frac{c^{2}}{w}+w<0
$$

because

$$
-c^{2}+w^{2}<0
$$

and $w$ is assumed to be a positive number. For very small $w / c$ we have:

$$
x_{1}=\frac{c}{w}\left[1-\left(1-\frac{w^{2}}{c^{2}}\right)^{1 / 2}\right] \approx 0
$$

and

$$
x_{2}=\frac{c}{w}\left[1+\left(1-\frac{w^{2}}{c^{2}}\right)^{1 / 2}\right] \approx 2 \frac{c}{w}
$$

therefore

$$
0<x_{1}<x_{\min }<x_{2} .
$$

A similar analysis can be done for the case when the function $F_{2}(x)$ is entering the difference (5) in place of $F_{1}(x)$, so $-w$ in $u^{\prime}$ is replaced by $w$. In this case we have in (5) [see (14)]:

$$
F_{2}(x)=x^{2} w+2 x c+w
$$

and

$$
F_{2}^{\prime}(x)=2 x w+2 c=0
$$

therefore

$$
x=x_{\min }=-\frac{c}{w} .
$$


In effect

$$
F_{2}\left(x_{\min }\right)=\frac{c^{2}}{w^{2}} w+2 c\left(-\frac{c}{w}\right)+w=-\frac{c^{2}}{w}+w
$$

which is the result identical to that obtained in (20).

As a sequence of solutions we obtain in the case of the function $F_{2}(x)$ a set due to $(16)$ and $(27):$

$$
x_{2}<x_{\min }<x_{1}<0 .
$$

\section{Stopping Velocity $w$ Taken into Account with Two Different Signs in Equation (6)}

Our aim is to calculate the size of the stopping velocity $w$ entering (6). At the first step we take $w$ with a negative sign. In this case it is evident from Equation (6) that we have the relation:

$$
(u-w)^{2}-u^{2}\left(1-\frac{u w}{c^{2}}\right)^{2}=0
$$

or

$$
\left(1-\frac{w}{u}\right)^{2}=\left(1-\frac{u w}{c^{2}}\right)^{2}
$$

which gives

$$
1-\frac{w}{u}= \pm\left(1-\frac{u w}{c^{2}}\right) .
$$

In the case of the positive sign on the right of (32) we obtain

$$
-\frac{w}{u}=-\frac{u w}{c^{2}}
$$

or

$$
c^{2}=u^{2}
$$

which is a result independent of the size and sign of $w$, moreover (34) is unacceptable for the physical reasons.

On the other hand, a minus sign before the brackets in (32) gives

$$
1-\frac{w}{u}=1-\frac{w}{c} \frac{c}{u}=-1+\frac{u w}{c^{2}}=-1+\frac{u}{c} \frac{w}{c},
$$

or

$$
2=\frac{w / c}{u / c}+\frac{u}{c} \frac{w}{c},
$$

which can be next transformed into

$$
2 \frac{u}{c}=\frac{w}{c}\left(1+\frac{u^{2}}{c^{2}}\right) .
$$

This gives

$$
w=\frac{2 u}{1+\left(\frac{u}{c}\right)^{2}} .
$$

Evidently a replacement of $-w$ in (30) by $w$ gives instead of (36) the result

$$
w=-\frac{2 u}{1+\left(\frac{u}{c}\right)^{2}} .
$$

In effect the sign of $w$ depends on the sign of $u$ : the signs of both $w$ and $u$ should be the same for the case of $-w$ substituted into $u^{\prime}$ entering the formula (6). But $w$ should have an opposite sign to that of $u$ in the case when $w$ with a positive sign does enter $u^{\prime}$ in (6). 


\section{Summary}

The problem raised in the paper concerns the cancellation of the particle kinetic energy obtained as an effect of the presence of a suitable velocity $w$ of the motion frame. This relativistic problem can be easily solved on condition the formulae concerning the original particle velocity $u$ and its modification due to the velocity $w$ are applied. In effect, we obtain a set of equations which couple the speed $u$ with the speed $w$. In practice, the quadratic equations for $u$ expressed in terms of $w$ and the light velocity $c$ are presented and discussed. A separate point was to calculate the extremal values of the speed $w$ required to cancel the difference between the square values of a modified and original particle velocity $u$.

First, we note that cancellation of the kinetic energy of a moving particle can be obtained from the equation

$$
u^{\prime 2}-u^{2}=0,
$$

where $u^{\prime}$ is the particle velocity modified by the motion of the system frame, and $u$ is the original (unmodified) particle velocity.

Next we consider (38) satisfied in two ways. In the first one (case A) the velocity $u$ is modified by the velocity $w$ of the system frame taken with a minus sign. Another way of modification of $u$ (case B) is done by the velocity $w$ taken with a positive sign. Both cases-A and Blead to solution pairs of $x_{1}$ and $x_{2}$ represented with the aid of the ratios

$$
\frac{u}{c}=x \text {. }
$$

In the case of $\mathrm{A}$ the solution pair $x_{1}$ and $x_{2}$ of (38) is obtained negative; on the other hand, the case of $\mathrm{B}$ provides us with a positive pair of $x_{1}$ and $x_{2}$.

A characteristic point is that both pairs of $x_{1}$ and $x_{2}$ are defined with the aid of a real value of the radical

$$
\sqrt{1-\frac{w^{2}}{c^{2}}}
$$

This expression, containing the stopping velocity $w$ is entering solely as a result of calculations: it has not been introduced as an effect of the physical background of the relativistic theory.

At the end of Section 4, we show that reduction of the particle velocity can be attained by the velocity direction of the motion frame being opposite to the velocity possessed by the particle.

\section{Conflicts of Interest}

The author declares no conflicts of interest regarding the publication of this paper.

\section{References}

[1] Sommerfeld, A. (1949) Mechanik. 4th Edition, Akademische Verlagsgesellschaft Geest \& Portig, Leipzig.

[2] Landau, L.D. and Lifshits, E.M. (1948) Field Theory. OGIZ, Moscow. (In Russian)

[3] Jauncey, G.E.M. (1948) Modern Physics. 3rd Edition, Van Nostrand, New York. 